\title{
Look little, look often: The influence of gaze frequency on drawing accuracy
}

\author{
DALE J. COHEN \\ University of North Carolina, Wilmington, North Carolina
}

\begin{abstract}
The present article attempts to determine what those who draw accurately do differently than those who do not. Four experiments explore the relation between drawing accuracy and the rate at which artists glance between their drawing and the stimulus (termed gaze frequency). Experiment 1 revealed a positive relation between gaze frequency and drawing accuracy $\left(r^{2}=.33\right)$. Experiments 2,3 , and 4 demonstrated that gaze frequency directly influences drawing accuracy. High gaze frequencies may facilitate drawing accuracy by (1) allowing the artist to hold less information in working memory, (2) reducing memory distortion, and (3) facilitating the reduction of context effects through inattentional blindness.
\end{abstract}

Drawing is a complex procedure that recruits numerous cognitive, perceptual, and motor processes, ranging from those involved in the initial viewing of the stimulus to those involved in the evaluation of one's rendering (D. J. Cohen \& Bennett, 1997). A breakdown in one or more of these processes can lead to drawing errors, so it is not surprising that most people cannot draw what they see. Nevertheless, some people succeed at drawing what they see. This article attempts to determine what those who succeed do differently than those who fail.

\section{Drawing Accuracy and Stimulus Interpretation}

In the year 415, Wang Wei hypothesized that adults' drawing errors arise as a result of perceptual transformations. He stated that young artists should be wary of their perceptions of objects because "the form of the object must first fuse with the spirit, after which the mind transforms it in many ways" (Sze, 1956, p. 39). In the 19th century, John Ruskin popularized this hypothesis and proposed that the perceptual transformations that lead to drawing errors are the result of learned associations. Ruskin coined the term the innocent eye to describe the act of viewing stimuli without the contamination of learned associations (Ruskin, 1843, cited in Rosenberg, 1963). Although Ruskin believed that one could not realistically render an object without acquiring an innocent eye, he believed that a truly innocent eye is unattainable.

The influence of stimulus interpretation on the drawing process is relatively well documented (Blakemore, 1973; Blakemore, Carpenter, \& Georgeson, 1970; Deregowski, 1973; Freeman, 1980, 1987; Gregory, 1990; Lee, 1989; Reith, 1988; Van Sommers, 1984; Willats, 1997). Van Sommers provided a direct demonstration of this influ-

Correspondence should be addressed to D. J. Cohen, Department of Psychology, University of North Carolina, Wilmington, NC 28403-3297 (e-mail: cohend@uncw.edu). ence by creating simple line drawings that had two viable interpretations (see Figure 1). The author presented participants with each line drawing along with one of its interpretations and asked them to copy the line drawings. Van Sommers videotaped the participants' executions of the drawings. The data revealed that their interpretations of the stimuli influenced their stroke direction and position. For example, when presented with the stimulus in Figure 1, participants who were told that it represented crossed swords drew two straight lines that crossed in the middle. In contrast, those who were told that it represented sniffing mice drew angled lines that represented each mouse's nose.

More generally, Lee (1989) has demonstrated that stimulus interpretation will negatively influence the drawing process even when the stimulus is unambiguous. Lee presented black and white outline drawings of parts of tables to primary and secondary school children and asked them to copy the stimuli exactly as they were presented. In one experiment, the children were told that the stimuli represented parts of a table. In another experiment, they were shown the same stimuli, but they were not told what the stimuli represented. The data showed that the children who interpreted the stimuli as parts of tables made large and robust drawing errors. In contrast, those who were given no interpretation of the stimuli made few drawing errors. Thus, Lee provided a convincing demonstration that stimulus interpretation influences drawing accuracy even in the absence of ambiguity.

Although stimulus interpretation is one source of drawing errors, many stages in the drawing process may also contribute to drawing errors. D. J. Cohen and Bennett (1997) assessed the influence of stimulus perception on adults' drawing errors relative to other probable sources. The authors divided the drawing process into four broad stages and assessed each stage's contribution to drawing errors. The first stage encompasses the perceptual processes involved in perceiving the to-be-drawn stimulus 


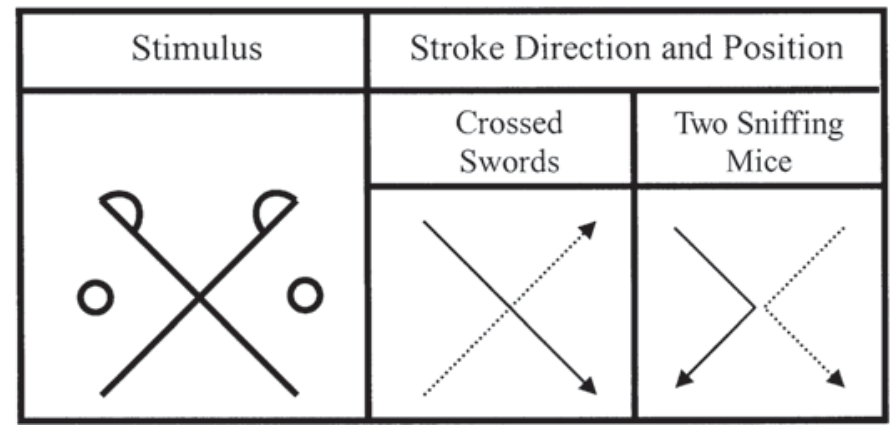

Figure 1. An example of a stimulus used in Van Sommers's (1984) research. Participants who were told that the stimulus represented crossed swords drew two straight lines that crossed in the middle. Participants who were told that the stimulus represented sniffing mice drew angled lines that represented each mouse's nose.

(e.g., stimulus interpretation). The second stage encompasses the decision processes involved in determining what information to represent and how to represent it. The third stage encompasses the motor processes involved in physically making the mark, and the final stage encompasses the perceptual and cognitive processes involved in evaluating the accuracy of one's rendering. In a series of experiments, D. J. Cohen and Bennett demonstrated that most of adult drawing errors are not the result of motor processes, decision processes, or the processes involved in evaluating the accuracy of one's rendering, but had their foundation in the initial perceptual stage. ${ }^{1}$

If the initial perceptual stage is the largest contributor to drawing errors, overcoming the difficulties associated with that stage would be critical to accurately rendering a stimulus. Therefore, those who can accurately render a stimulus (e.g., trained artists) should be less susceptible to the illusions associated with the perception (or misperception) of stimuli. Thouless (1932) directly tested this hypothesis by assessing whether trained artists could overcome the influence of stimulus interpretation by using a test of shape constancy (which Thouless termed phenomenal regression). Shape constancy refers to the phenomenon that obliquely presented shapes are perceived as less skewed than the shape projected on one's retina. For example, an obliquely presented circle projects an ellipse on the retina, yet people perceive the ellipse as more circular than its retinal projection. The common explanation of this phenomenon is that one's knowledge of the true shape of the object influences his or her perceived shape of the object. Thouless demonstrated that trained artists are less susceptible to the biases induced by shape constancy than the average observer.

Recently, Kozbelt (2001) argued that trained artists' superior visual cognition skills are not limited to shape constancy. Kozbelt measured nonartists and art students' abilities to accurately draw various stimuli, identify the subjects of blurred and incomplete pictures, find visually camouflaged objects, and complete a mental rotation task. The data revealed that art students outperformed nonartists on virtually all tasks. Kozbelt's analysis also revealed high correlation between the performance on the perception tasks and that on the drawing tasks.

\section{Drawing Accuracy and Gaze Frequency}

Although there is substantial evidence that artists are better able to accurately analyze visual stimuli than nonartists, and that this ability is correlated with the ability to render more realistic drawings, the process through which artists accomplish this feat has not been well studied. The present article is an attempt to identify an observable behavior that directly contributes to drawing accuracy. I present four experiments that explore the relation between drawing accuracy and the rate at which artists glance between their drawing and the stimulus (termed gaze frequency). Here, gaze frequency is measured in hertz and is operationally defined as the number of times per second that an artist switches his or her gaze from the stimulus to the drawing and back again. Thus, a high gaze frequency refers to relatively fast alternations between the drawing and the stimulus, and a low gaze frequency refers to relatively slow alternations between the drawing and the stimulus. I focus on this behavior for two reasons. First, there is some evidence that drawing accuracy is related to gaze frequency (Miall \& Tchalenko, 2001), though this evidence is anecdotal and preliminary. Second, this behavior is both directly observable and controllable, thus lending itself to experimental manipulation.

The anecdotal evidence supporting the influence of gaze frequency on drawing accuracy comes from statements by artists. For example, Edgar Degas stated that were he to start an art school, he would have the model and students situated on separate floors (Kendall, 1993). Presumably, this would force the students to commit significant aspects of the stimulus to memory before drawing them. JeanAuguste-Dominque Ingres, one of the most acclaimed western realists, agreed with Degas. He extorted artists to "Never work from nature. Always from memory..." (Kendall, 1993, p. 210). A strategy of committing significant aspects of the stimulus to memory before drawing would 
presumably require long fixation times (i.e., a low gaze frequency), thus leading to a negative relation between drawing accuracy and gaze frequency.

An extensive case study by Miall and Tchalenko (2001) provides some support for the negative relation between drawing accuracy and gaze frequency. Miall and Tchalenko tracked an accomplished artist's (Humphrey Ocean) eye movements over the course of creating several portraits. They found that his average fixation was about $1 \mathrm{sec}(0.5 \mathrm{~Hz})$. They also tracked the eye movements of three novice artists drawing a 1-min portrait. The average fixation of the novice artists was approximately half that of Ocean $(0.25 \mathrm{~Hz})$. The authors conclude that

It seems plausible according to this data that a graded pattern of eye movements correlates with drawing skills. The most fluid and accurate drawing by our three novices was made with the most consistent, longest fixations on the model, the least accurate, with the least consistent eye movements. However, more tests are needed to confirm this. (p. 38)

In contrast to the views of these select artists and the findings of Miall and Tchalenko (2001), the literature addressing eye movements in natural tasks suggests that a positive relation should exist between drawing accuracy and gaze frequency. In particular, Ballard, Hayhoe, and Pelz (1995) provide evidence that people naturally use frequent shifts in eye fixations to compensate for the limited capacity of working memory (WM). Ballard et al. (1995) state that human memory representations result from a deictic system, which stores pointers to the location of perceptual information in the visual scene rather than storing the perceptual information directly. Such a system minimizes the use of WM by postponing the gathering of relevant perceptual information until it is required. Ballard et al. (1995) demonstrate that participants' eye fixations in a block-pattern copying task are consistent with this interpretation.

A deictic system predicts that accomplished artists will store small amounts of visual information just prior to drawing that information, a strategy that will result in a high gaze frequency. A deictic system also predicts that reducing gaze frequency will force artists to increase the amount of information in WM, which in turn will result in poor memory representations and thus poor drawings. If a deictic system is an inherent part of the human memory system, the drawings of both trained and novice artists would be negatively affected by low gaze frequencies.

The present article assesses the relation between gaze frequency and drawing accuracy. Experiment 1 assesses the correlation between gaze frequency and drawing accuracy. Experiments 2, 3, and 4 assess the causal relation between gaze frequency and drawing accuracy.

\section{EXPERIMENT 1}

The participants in Experiment 1 were presented, in succession, with two color photographs and asked to realistically render the images in the photographs. They were allowed $10 \mathrm{~min}$ to complete each drawing. A video camera recorded the participants' eye and head movements. Because it has been claimed that presenting to-bedrawn stimuli upside down increases drawing accuracy (Edwards, 1999), half of the photographs were presented upside down. To ensure a wide range of drawing abilities, both art majors and nonart majors were tested. Drawing accuracy was rated by 30 nonart major students.

\section{Method}

\section{Participants}

Artists. Twelve art majors and 12 nonart majors volunteered to participate as artists. Here, the term artist refers to those participants who draw the stimulus. The term, as used here, implies no training or skill level. Throughout the paper, the art majors are referred to as trained artists, and the nonart majors are referred to as novice artists.

Critics. Thirty-one participants volunteered to participate as critics. Here, the term critic refers to those participants who rated the accuracy of the drawings. The critics had no formal training in the visual arts.

Because there is no universal computer algorithm for comparing the accuracy of renderings with that being rendered, one must resort to using critics to judge the accuracy of a rendering. Furthermore, although one may be tempted to state that a photograph is the epitome of depiction, two prints created from the same negative can look very different.

One can choose to recruit either expert or novice critics to judge the accuracy of renderings. The difficulty with recruiting experts to judge the accuracy of a rendering is fourfold. First, it is unclear on what objective basis one defines an expert. Second, even experts from the same profession do not have a uniform definition of accuracy. A third problem with experts is that they are very different from the participants in this experiment. Thus, their objectives may be very different from those of the participants. As such, if the artist achieved his or her intent perfectly and the artist's intent is different from that of the expert critic, the critic's rating of accuracy will be in conflict with the artist's assessment. Finally, expert critics are difficult to come by. Therefore, one cannot gather a large enough group of experts, such that the personal bias of any one expert will have a minimal effect on the average rating.

A second option is to recruit novice critics from the same population from which the artists are recruited. This option has several advantages. First, these critics are numerous, so relatively large numbers can be recruited. Second, the large numbers will reduce the personal biases of any individual critic. Third, because both artists and critics come from the same population, the probability of congruous intents is heightened. The one disadvantage is that these critics have no recognized expertise in the area. Despite the lack of recognition, several studies have indicated that people in general are expert picture perceivers (for a review, see Hochberg, 1996). Finally, although artists and critics are affected by the same cognitive biases, these biases tend to affect one's ability to draw the stimulus far more than one's ability to judge the accuracy of the stimulus ${ }^{2}$ (Cohen \& Bennett, 1997, Experiment 4).

To reiterate, because there is no universal computer algorithm for comparing the accuracy of renderings with that being rendered, people must judge the accuracy of the renderings. In addition, there is no overriding advantage to using either expert or novice critics. Because I believe that large numbers of critics with similar intents to those of the artists is important, I made the decision to use novice critics.

\section{Materials}

Two $20.3 \times 25.4 \mathrm{~cm}$ color photographs were used as stimuli in the rendering task. The two stimuli were photographic portraits of the 
heads of males seen from the shoulders up. One of the models was dark skinned, and the other was light skinned. The photographs were placed in a $24.1 \times 4.3 \mathrm{~cm}$ transparent page protector and clipped to a black backboard. The backboard was placed $45^{\circ}$ clockwise (relative to perpendicular to the participant) and approximately $51 \mathrm{~cm}$ to the right of the participant. The position of the backboard allowed an unobstructed view for a video recorder, which was positioned $122 \mathrm{~cm}$ directly in front of the participant.

Faces were chosen as stimuli for several reasons. First, my colleague and I have shown that participants draw upright color photographs of faces as accurately as they draw upright color photographs of a generator in a variety of experimental procedures (D. J. Cohen $\&$ Bennett, 1997). Thus, in a rendering task, faces are likely no different from other complex stimuli. Second, there is extensive literature addressing perception of upside-down faces (e.g., Diamond \& Carey, 1986; Leder \& Bruce, 2000; Murray, Yong, \& Rhodes, 2000; Tanaka \& Sengeo, 1997). Therefore, any differences in drawing accuracy found in the present experiment can be related to that literature, the likes of which do not exist for other stimuli. Third, because Miall and Tchalenko (2001) used faces as stimuli in their study of portrait painter Humphrey Ocean, the results of the present experiment will be comparable to those of Miall and Tchalenko's study. Finally, it is common practice for art professors to teach students to render portraits (i.e., faces) upside down. Therefore, the assessment of drawing that uses these externally valid stimuli carries applied implications that using abstract or simplified stimuli (e.g., ellipses) would not

The video recorder was placed in the doorway of an adjoining room. A curtain covered the doorway, and a hole was cut in the center of the curtain for the camera lens. The participants were told that they were being video recorded. The presence of the video recorder lens did not seem to affect the participants' performance.

In the rendering task, the participants used a No. 2 pencil on white typing paper. The critics were provided with the original photographs and the participants' renderings. These stimuli were placed in transparent page protectors to increase durability and arranged in a three-ring binder.

\section{Procedure}

The artist's task. All the artists were tested individually. Each artist drew both photographs, one of which was presented rightside up and the other upside down. The order of presentation was counterbalanced. The instructions read to the artist were identical to those used by D. J. Cohen and Bennett (1997). In short, the artist was asked to draw the photograph as visually accurate as possible. Visual accuracy was explained as photo realism (given the limits of the medium). The artist was further instructed that only the visual accuracy of the rendering was important and that aesthetics, style, and creativity were not valued. Aesthetic value, style, and creativity were explained, through the use of verbal examples, as creative abstractions similar to those of Picasso or Matisse. All the artists indicated that they understood the task.

Each artist was given $10 \mathrm{~min}$ to render each photograph. The experimenter presented the photograph, told the artist to begin, and then left the room for exactly $10 \mathrm{~min}$. The artist was told to stop drawing when the experimenter reentered the room. The 10-min allotment was sufficient, since most artists indicated completion by that time (see D. J. Cohen \& Bennett, 1997). This procedure was repeated for each photograph. At the completion of the task, the artists were interviewed about their formal training in the visual arts.

The critic's task. The renderings of the photographs produced by the artist were used in the critic's task ( 24 renderings of each photograph). Each critic was presented with the original photographs and a binder containing the 48 renderings produced by the artists. The order of the artists' renderings was randomized among critics. All the critics were tested individually.

The critic was told to rate the rendering's visual accuracy on a scale from 1 to 10, with 1 indicating a very poor representation and
10 indicating a very accurate representation. The critic was given the same explanation of visual accuracy as were the participants. The critic was permitted to take as much time as he or she needed to assess each rendering. All of the critics indicated that they understood the task.

Coding eye movements. I wrote a computer program to help code the participants' eye movements. Three keys were activated on the computer keyboard, one to indicate that the artist was viewing his or her rendering, one to indicate that the artist was viewing the photograph, and one to indicate that the artist was not looking at either the drawing or the photograph. The coder watched the videotapes of each artist's performance, pressing the appropriate keys as the artist switched his or her eye gaze. The computer recorded the key that was pressed and the time in milliseconds between keypresses. Because the video camera was positioned as a close-up head shot of the artist, his or her eye gaze switches were clearly visible. In addition, because all artists were referred to by an arbitrary number, and the artist's renderings were not visible in the videotape, the coder was blind to the artist's skill level.

\section{Results}

\section{Reliability of Coding Eye Movements}

The use of manual coding of eye gaze switches, rather than an eyetracker, presents two difficulties. First, the amount of information obtained from the data is reduced, relative to that obtained from an eyetracker. For this reason, my analysis focuses solely on gaze frequency, rather than on the precise spatial location of gaze fixations. Second, manual coding of eye gaze switches introduces noise into the data. In particular, the coder's reaction time to the movement of the switch is variable, and that variability cannot be separated from the variability associated with the artists' eye gaze switches. Nevertheless, the coding variability should be random (i.e., unrelated to drawing accuracy). Therefore, the noise introduced by the coder will have the result of simply reducing the power of the analysis. For this reason, any effects observed in the data are likely underestimated.

To assess the reliability of the data, a random subset of the data was selected for recoding by an independent coder, and the timing data from the two coders were correlated. Because a single spurious point can dramatically reduce the correlation between the two data sets, the data were examined for spurious points. A spurious point is indicated by (1) more eye gaze switches recorded by one of the coders than the other, and (2) the double coding of a gaze location (e.g., two successive codings of the artist gazing at the drawing). A maximum of three points were removed in each data set, approximately $0.7 \%$ of the data (or about 3 out of 400 data points). The correlation between the data from the two coders averaged $r=$ $.98(S D=.001)$. Furthermore, the average gaze duration recorded by the two coders differed on average by only $10 \mathrm{msec}(S D=11 \mathrm{msec})$. Thus, the manually coded data were reliable.

\section{Reliability of Critics' Ratings}

Novice critics were selected, in part, because a large enough sample could be gathered so any individual critic would not have too much influence on the data. Nevertheless, it is important to assess the reliability of the critics' 


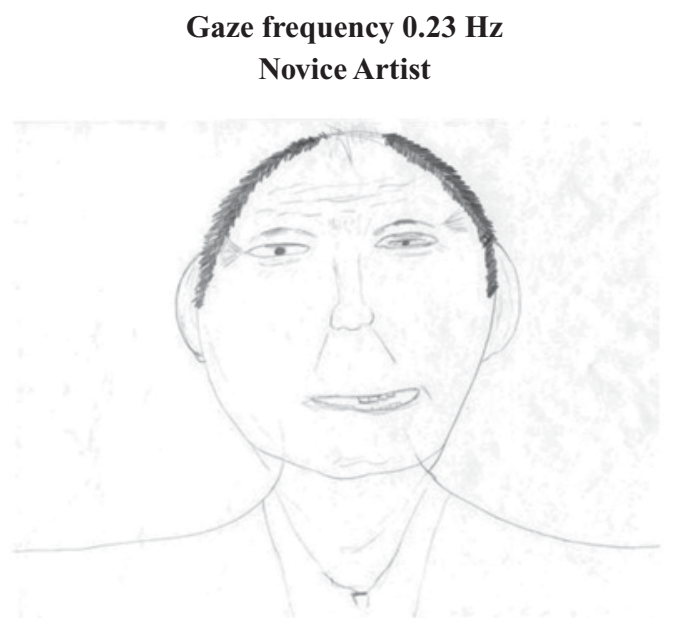

\section{Gaze frequency $0.5 \mathrm{~Hz}$ \\ Trained Artist}

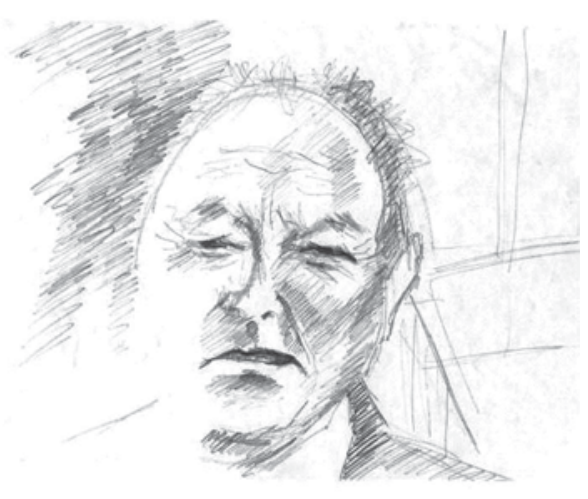

Figure 2. Examples of a low- and a high-rated rendering from Experiment 1.

ratings. Cronbach's alpha $(\alpha=.97)$ showed that, although novice critics were selected, their ratings were remarkably reliable, given the subjective nature of the task (a value greater than .7 is considered good reliability; Nunnaly, 1978). The high reliability is, in part, a function of the range of drawing accuracy exhibited by the artists (see Figure 2).

\section{Analysis of Eye Movements}

The critics' mean rating for each rendering was calculated and used as the dependent variable. To assess the effect of photograph (Face A vs. Face B), position (upside down vs. rightside up), and artist training (novice vs. trained) on drawing accuracy, a $2 \times 2 \times 2$ mixed ANOVA was calculated. As expected, there was a significant effect of artist training $[F(1,22)=110, p<.01]$, in which the renderings of trained artists $(M=5.58, S D=0.87)$ were rated as more accurate than those of the novice artists $(M=2.74, S D=0.61)$. There were no other significant effects (all $F \mathrm{~s}<1.0$ ). Because there was no effect of photograph or position, the data were collapsed over these variables for all other analyses.

To assess the relation between eye movements and drawing accuracy, a hierarchical regression was calculated using total drawing time, photo/drawing ratio, and gaze frequency as predictor variables and critics' mean rating for each rendering as the criterion variable. Total drawing time was time in milliseconds that the artist spent drawing each photograph. Although the artists were told to participate for $10 \mathrm{~min}$, the videotapes revealed some variation in participation times $(M=10.2 \mathrm{~min}, S D=$ $0.27 \mathrm{~min}$ ). This variable was entered first into the regression to remove any effect of participation time, presuming that an effect existed. Photo/drawing ratio was the time spent viewing the photograph divided by the time spent viewing his or her drawing $\left(T_{\text {photo }} / T_{\text {drawing }}\right)$. This variable, which indicates how the artist divided up his or her time during the session, was included to assess whether those who draw accurately divide up their time differently than those who do not. If the artist viewed the photo and the drawing equally, this variable would equal one. A value greater than one indicates that the artist viewed the photo more often than the drawing, and a value less than one indicates that the artist viewed the photo less often than the drawing $(M=0.45, S D=0.18)$. This variable was entered second into the regression. To provide a strong test of the hypothesis that gaze frequency is related to drawing accuracy, gaze frequency was entered last into the regression. Gaze frequency is measured in hertz and therefore indicates the average number of times the artist switched his or her gaze from the photo to the drawing and back again per second $[M=0.33$ (1.5-sec fixations), $S D=$ $0.11]$. Although all of these variables are theoretically independent, photo/drawing ratio and gaze frequency are correlated $(r=.66$; see Table 1 for a full correlation matrix). This indicates that these two variables share about $43.5 \%$ of their variance. Because gaze frequency is entered into the regression last, it is mathematically handicapped when predicting drawing accuracy.

The hierarchical regression was significant $[F(3,44)=$ 7.2, $p<.01, r^{2}=.33$ ]. The only significant predictor was gaze frequency. ${ }^{3}$ Specifically, there was a positive relation between drawing accuracy and gaze frequency (slope $=9.86, t=4.03, p<.01$; see Figure 3 ). Neither the intercept nor the remaining two predictors were significant (all $t \mathrm{~s}<1.2$ ). Because photo/drawing ratio was (1) highly correlated with gaze frequency, (2) entered into the regression before gaze frequency, and (3) found to be

Table 1

Correlations ( $r$ ) Among Drawing Accuracy, Total Drawing Time, Photo/Drawing Ratio, and Gaze Frequency

\begin{tabular}{lccc}
\hline & $\begin{array}{c}\text { Drawing } \\
\text { Accuracy }\end{array}$ & $\begin{array}{c}\text { Gaze } \\
\text { Frequency }\end{array}$ & $\begin{array}{c}\text { Photo/Drawing } \\
\text { Ratio }\end{array}$ \\
\hline Total drawing time & .17 & .21 & .21 \\
Photo/drawing ratio & .26 & .66 & \\
Gaze frequency & .55 & & \\
\hline
\end{tabular}




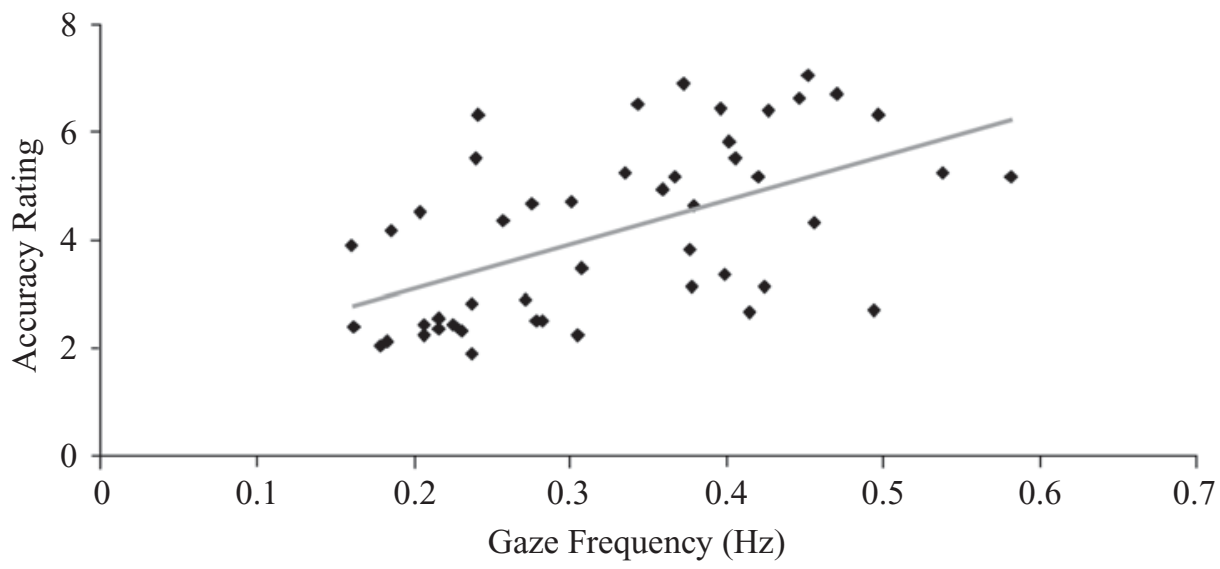

Figure 3. The relation between drawing accuracy and gaze frequency. Gaze frequency accounted for $33 \%$ of the variance associated with drawing accuracy.

a nonsignificant predictor, the present analysis reveals that the variance in drawing accuracy accounted for by gaze frequency was independent of photo/drawing ratio.

In accord with the results of the regression, there was no significant effect of artist training on photo/drawing ratio $[t(46)=0.61$, n.s. $]$, and there was a significant effect of artist training on gaze frequency $[t(46)=3.9, p<.01]$, in which the gaze frequencies of trained artists $[M=0.38$ (1.3-sec fixations), $S D=0.1]$ were greater than those of the novice artists $[M=0.27$ (1.85-sec fixations), $S D=0.09]$.

Both trained and novice artists were included in the present experiment to ensure a range of accuracy ratings large enough to detect an effect of gaze frequency, if one existed. Nevertheless, when a regression is calculated on two groups that differ on a variable of interest, a spurious correlation can result. To test for this, I calculated separate correlations on each group. Unfortunately, this procedure results in a truncated range of accuracy ratings within each group, thus unavoidably reducing the expected correlation (J. Cohen \& P. Cohen, 1983). As expected, the correlation between drawing accuracy and gaze frequency declined from $r=.55$ in the entire data set to nonsignificant, though positive, values of $r=.24$ for novice artists and $r=.25$ for trained artists. The nonsignificant values were likely a result of a single artist whose data appear as an outlier when the data are split into two groups. This participant's data are not an outlier in either the overall or trained artists' data sets, suggesting that he or she performed more like the trained artists than the novice artists (his or her mean accuracy was 4.0). When this participant is shifted from the novice artists group to the trained artists group, the correlations for both groups raise considerably to $r=.63(p<.01)$ for novice artists and $r=.46$ $(p=.01)$ for trained artists (see Figure 4). Thus, although trained artists in general drew more accurately than novice artists, a positive relation between drawing accuracy and gaze frequency exists for both groups.

\section{Discussion}

The data demonstrate that a relation exists between drawing accuracy and gaze frequency. In particular, the higher the artist's gaze frequency, the greater his or her drawing accuracy. This result is particularly informative because gaze frequency accounted for $33 \%$ of the variance in drawing accuracy, which suggests that gaze frequency is an important contributor to drawing accuracy. In addition, neither cumulative drawing time nor how the artists distributed their gaze time between the photograph and the drawing were related to drawing accuracy.

A surprising finding that I do not pursue in the present article is that the position of the photograph (upside down vs. rightside up) does not influence drawing accuracy. This result is surprising because positioning a photograph upside down is a relatively common practice in art schools. Professors of art will turn photographs upside down with the intention of inhibiting stimulus interpretation, which in turn is intended to increase the accuracy of the students' drawing. The result of the present experiment suggests that any benefit of turning a photograph upside down may have more to do with expectations than perceptual changes. Nevertheless, because faces were used as stimuli and the literature demonstrates a robust faceinversion effect, further research must be completed to determine whether this result generalizes to other stimuli. Because there was no effect of photograph position, this variable was not manipulated in Experiments 2-4 (i.e., the photographs were only presented rightside up in the remaining experiments).

The result that a positive relation exists between drawing accuracy and gaze frequency appears to disconfirm Degas's proposed training technique of positioning the model and student on different floors. However, because this experiment only assessed the relation between drawing accuracy and gaze frequency, no causal conclusions can be reached. In Experiment 2, I assess whether manipulating gaze frequency will influence drawing ability.

\section{EXPERIMENT 2}

Experiment 2 was designed to assess whether gaze frequency influences drawing ability. An apparatus was created in which participants could alternately see their 
Trained Artists

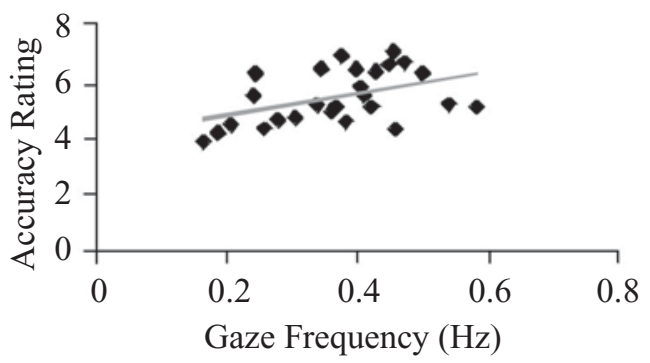

Novice Artists

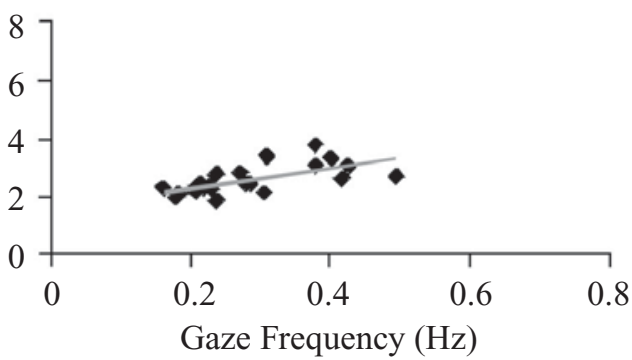

Figure 4. The relation between drawing accuracy and gaze frequency for trained and novice artists.

drawings or the to-be-drawn photograph. The speed of alternation between the drawing and photograph was experimentally manipulated.

\section{Method}

\section{Participants}

Ten art majors (i.e., trained artists) and 24 nonart majors (i.e., novice artists) volunteered to participate as artists. Thirty nonart majors volunteered to participate as critics. The critics had no formal training in the visual arts.

\section{Apparatus}

Three photographs were used as stimuli in the rendering task. They included the two photographs used in Experiment 1 and an additional photograph of a light-skinned female.

An apparatus was created to alternately reveal the photograph and the drawing. It consisted of two boxes: one to house the photograph and one to house the drawing (see Figure 5). The dimensions of the box housing the photograph were $60 \times 60 \times 30.5 \mathrm{~cm}$ (height $\times$ width $\times$ depth). In the center of one large face of the box was a $22 \times 28 \mathrm{~cm}$ hole, which was covered by tightly woven insect screen mesh. The photograph was placed $30.5 \mathrm{~cm}$ behind the mesh. A 40-W light bulb was wired inside the box. The mesh provided an effective visual screen, such that the photograph was visible only when the light inside the box was turned on. When the light was turned off, nothing behind the mesh was visible.

The dimensions of the box housing the drawing were $25.5 \times$ $60 \times 60 \mathrm{~cm}$. A $22 \times 28 \mathrm{~cm}$ hole, cut $2.5 \mathrm{~cm}$ from the leading edge of the large face of the box, was covered by tightly woven insect screen mesh. The paper for the drawing was placed $25.5 \mathrm{~cm}$ behind the mesh (i.e., resting on the table). A second hole measuring $10 \times$ $27 \mathrm{~cm}$ was cut from the leading face of the box to allow the artist's hand and arm access to the paper that was contained within the box. A 40-W lightbulb was wired inside the box. The mesh provided an effective visual screen, such that the drawing was visible only when the light inside the box was turned on. When the light was turned off, nothing behind the mesh was visible.

The box housing the drawing was placed so that the large face of the box was parallel to the table. The box housing the photograph was placed so that the large face of the box was perpendicular to the table and $165 \mathrm{~cm}$ directly in front of the box housing the drawing. A timing box, which linked the lights in the two boxes, alternately switched on and off the lights in the two boxes, so only one light was on at any one time. The box was wired so that the alternation time could be varied.

The room was illuminated by a single $60-\mathrm{W}$ lightbulb. This provided an ambient light level that ensured the invisibility of the

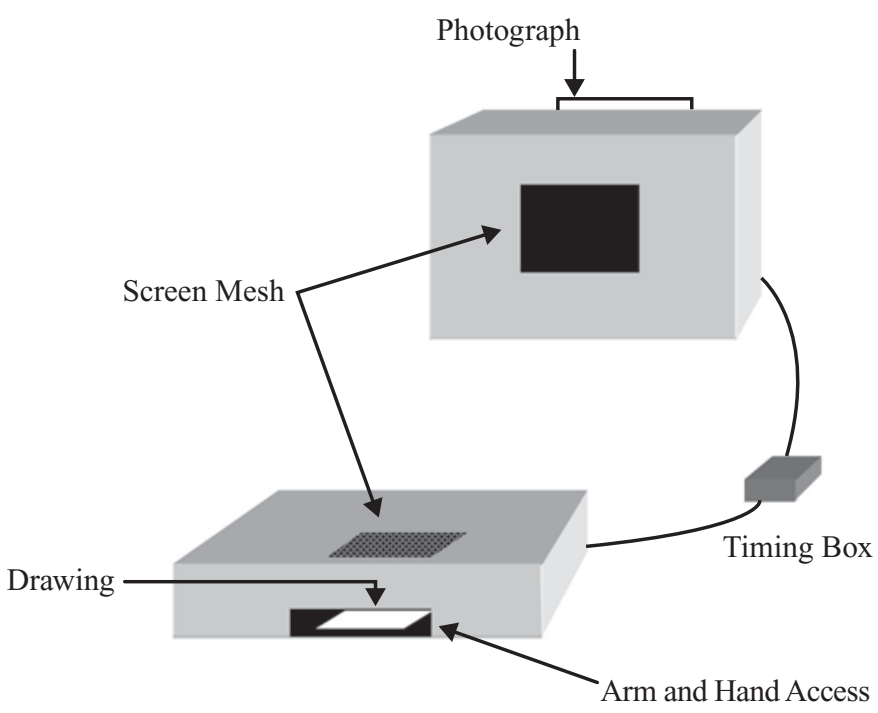

Figure 5. The apparatus used in Experiments 2-4. 
Table 2

Means and Standard Deviations of Drawing Accuracy Ratings By Artist Training and Gaze Frequency Condition From Experiment 2

\begin{tabular}{|c|c|c|c|c|c|c|}
\hline \multirow{3}{*}{$\begin{array}{c}\text { Gaze } \\
\text { Frequency }(\mathrm{Hz})\end{array}$} & \multicolumn{4}{|c|}{ Artist Training } & & \\
\hline & \multicolumn{2}{|c|}{ Trained } & \multicolumn{2}{|c|}{ Novice } & \multicolumn{2}{|c|}{ Average } \\
\hline & $M$ & $S D$ & $M$ & $S D$ & $M$ & $S D$ \\
\hline 0.034 & 5.07 & 1.53 & 3.29 & 1.05 & 3.81 & 1.45 \\
\hline 0.1 & 5.67 & 0.92 & 3.49 & 1.26 & 4.13 & 1.53 \\
\hline 0.5 & 6.18 & 1.36 & 3.39 & 1.36 & 4.21 & 1.86 \\
\hline Average & 5.64 & 1.21 & 3.38 & 1.32 & & \\
\hline
\end{tabular}

stimuli when the lights in the boxes were off and the visibility of the stimuli when the lights in the boxes were on. A video recorder, which was placed to the side of the box housing the photograph, recorded the eye and head movements of the artists.

In the rendering task, the artists used a No. 2 pencil on white typing paper. Their renderings were placed in transparent page protectors to increase durability and arranged in a three-ring binder along with the original photographs. The critics were given the three-ring binder when they were rating the accuracy of the photographs.

\section{Procedure}

The artist's task. Gaze frequency was manipulated by varying the timing of the light switches within the boxes. The lights in the boxes alternated every 1,5 , or $15 \mathrm{sec}$ (frequencies of $0.5,0.1$, and $0.034 \mathrm{~Hz}$, respectively, where $1 \mathrm{~Hz}$ equals one full cycle, photo light on followed by drawing light on, per second). These speeds were chosen for several reasons. First, the $0.5-\mathrm{Hz}$ frequency was chosen because it was one of the highest average gaze frequencies that an artist exhibited in Experiment 1 (see Figure 1). Second, the 0.1-Hz frequency was the lowest average gaze frequency of Experiment 1. Finally, the $0.034-\mathrm{Hz}$ frequency is the lowest gaze frequency that kept an entire cycle within the approximate limits of short-term WM (i.e., $30 \mathrm{sec}$; for a review, see Nairne, 1996). It is important to note that the only difference between conditions was the speed at which the lights alternated (i.e., the total time that the drawing and photograph were visible was constant across all three gaze frequency conditions). ${ }^{4}$ Gaze frequency was manipulated within artists. Each artist rendered each of the three photographs at a different gaze frequency. The photograph $\times$ gaze frequency combination was counterbalanced, as was the order of presentation.

All the artists were tested individually. Before drawing each photograph, the artist was given a 2-min practice period in which a blank piece of paper was substituted for the photograph. The artist was instructed to practice switching his or her eye gaze with the lights. After the practice period, the artist was given the same instructions as in Experiment 1. All artists indicated that they understood the task. After placing the photograph in the box used to house the photograph, the experimenter reset the timing box and left the room for exactly $10 \mathrm{~min}$. At the end of the 10 -min period, the timing box switched off the lights in both boxes. This procedure was repeated for each photograph. After completion of the task, the artist was interviewed about his or her formal training in the visual arts.

The critic's task. The renderings of the photographs produced in the artist's task were used in the critic's task. The critic's task was identical to that used in Experiment 1, except that the critics were presented with a binder containing 102 renderings, along with the original photographs. The order of the artists' renderings was randomized between the critics.

\section{Results}

The critics' mean rating for each rendering was calculated and used as the dependent variable (Cronbach's alpha, $\alpha=.97$; see Table 2). A $3 \times 2$ mixed-model repeated measures ANOVA was calculated on the accuracy ratings. In this analysis, artist was treated as a random effect. In accord with the proposed hypothesis, a contrast analysis assessed whether a linear relation exists between gaze frequency and drawing accuracy. The lambda weight for each gaze frequency condition equaled gaze frequency minus average gaze frequency $\left(\lambda_{i}=\mathrm{GF}_{i}-\mu_{\mathrm{GF}}\right.$, where GF is gaze frequency). There was a significant linear effect of gaze frequency $[F(1,64)=6.65, p<.01]$ and also a significant effect of artist training $[F(1,32)=30.37, p<$ $.01]$, in which the drawings of trained artists were rated as more accurate than those of novice artists. Finally, there was a significant interaction between artist training and gaze frequency $[F(2,64)=3.19, p<.05$; see Figure 5]. To clarify this interaction, contrast analyses that assessed whether a linear relation exists between gaze frequency and drawing accuracy were calculated on trained and novice artists separately. The analysis showed that trained artists displayed a significant linear effect of gaze frequency $[F(1,18)=6.37, p<.05]$. In contrast, novice artists displayed no effect of gaze frequency $[F(1,46)=0.02$, n.s.].

\section{Discussion}

The data demonstrate that gaze frequency influences drawing accuracy only for trained artists. Specifically, decreasing trained artists' gaze frequencies decreased their drawing accuracy. In contrast, gaze frequency had no effect on the drawing accuracy of novice artists. There are two likely reasons why there was no effect of gaze frequency for novice artists. First, after reviewing the video recordings of the artists, it became clear that the novice artists had trouble with the $0.5-\mathrm{Hz}$ frequency condition. The trained artists had no difficulty with this condition. Thus, it is possible that 1-sec alternations were too quick for the novice artists for follow. Second, any bounded scale will likely produce floor or ceiling effects if the average of the group is near one of the bounds. These effects are often evident in the data as a skew in the direction opposite of the bound. The critics' ratings of the novice artists' drawing accuracy were very near the floor of the scale and exhibited a positive skew. ${ }^{5}$ This suggests a floor effect. In contrast, the trained artists' mean accuracy rating was near the center of the scale and exhibited no skew. The floor effect exhibited in the novice artists' data may have been realized because the critics rated the novice and trained artists' drawings together. It is possible that the trained artists' drawings absorbed most of the scale, whereas the novice artists' drawings were relegated to the extreme low values. Experiment 3 addresses both of these issues.

\section{EXPERIMENT 3}

Experiment 3 addresses the shortcomings of Experiment 2. In particular, it replicates Experiment 2, with two exceptions. First, the gaze frequencies were lowered to 0.167 , 0.0625 , and $0.034 \mathrm{~Hz}$ (i.e., the lights in the boxes alternated every 3, 8, and $15 \mathrm{sec}$, respectively). Second, the critics rated the trained and novice artists' drawings separately. 


\section{Method}

\section{Participants}

Sixteen nonart majors were recruited to participate as novice artists, and 12 local artists were paid $\$ 10$ each to participate as trained artists. Sixty-six nonart majors volunteered to participate as critics; 33 of them rated the accuracy of the novice artists' drawings and 33 rated the accuracy of the trained artists' drawings. The critics had no formal training in the visual arts.

\section{Apparatus}

The same photographs and apparatus used in Experiment 2 were used in Experiment 3.

\section{Procedure}

The artist's task. The artist's task was identical to that of Experiment 2, except that the gaze frequencies were $0.167,0.0625$, and $0.034 \mathrm{~Hz}$ (i.e., the lights in the boxes alternated every 3,8 , and $15 \mathrm{sec}$, respectively).

The critic's task. The critic's task was identical to that of Experiment 2 , except that two spiral binders were created, one containing drawings by novice artists and one containing drawings by trained artists. The order of the artists' renderings was randomized between critics. To reduce floor effects in the novice condition, different critics rated the trained and novice artists. In this way, one could be confident that the trained artists' drawings would not influence the critics' ratings of the novice artists' drawings.

\section{Results}

To assess the effect of gaze frequency on drawing accuracy across artist training, drawing accuracy ratings were subjected to a $3 \times 2$ mixed model repeated measures ANOVA where artist was treated as a random effect (Cronbach's alpha, $\alpha=.95$ ). Because the trained and novice artists were rated separately, one cannot meaningfully compare the critics' mean ratings of the trained artists to those of the novice artists. Therefore, the main effect of artist training was not tested.

In accord with the proposed hypothesis, a contrast analysis assessed whether a linear relation exists between gaze frequency and drawing accuracy. The lambda weight for each gaze frequency condition equaled the gaze frequency minus the average gaze frequency $\left(\lambda_{i}=\mathrm{GF}_{i}-\mu_{\mathrm{GF}}\right.$, where
GF is gaze frequency). There was a significant linear effect of gaze frequency $[F(1,52)=5.88, p=.01$; see Figure 6], such that the drawings rendered in the $0.167-\mathrm{Hz}$ condition $(M=4.38, S D=1.04)$ were rated as more accurate than those rendered in the $0.0625-\mathrm{Hz}$ condition $(M=4.08$, $S D=1.26)$, which in turn were rated as more accurate than those rendered in the $0.034-\mathrm{Hz}$ condition $(M=3.93$, $S D=1.67)$. There was no significant interaction between artist training and gaze frequency $(F=1)$.

\section{Discussion}

The results of Experiment 3 replicate those of Experiment 2, except that the effect of gaze frequency is constant across artistic training level. The effect of gaze frequency likely emerged for the novice artists because the gaze frequencies were lower, thus allowing the novice artists to follow the light more accurately, and the floor effect associated with the drawing accuracy for novice artists was mitigated by having separate critics rate the accuracy of trained and novice artists' drawings.

Although Experiments 2 and 3 demonstrated that gaze frequency influences drawing accuracy, it was unclear whether this influence is facilitatory or inhibitory. In other words, the effect may have been found for one of two reasons: (1) High gaze frequencies increased drawing accuracy above the artists' baseline accuracy, or (2) low gaze frequencies decreased drawing accuracy below the artists' baseline accuracy. Because Experiment 1 demonstrated that artists' average gaze frequency was about $0.33 \mathrm{~Hz}$, and the highest successfully manipulated gaze frequency in Experiments 2 and 3 was $0.167 \mathrm{~Hz}$, it is likely that the effect of gaze frequency demonstrated in those two experiments was the result of inhibiting artists' drawing accuracy. Experiment 4 tests this hypothesis.

\section{EXPERIMENT 4}

Experiment 4 assesses whether gaze frequency facilitates or inhibits drawing accuracy as compared with the

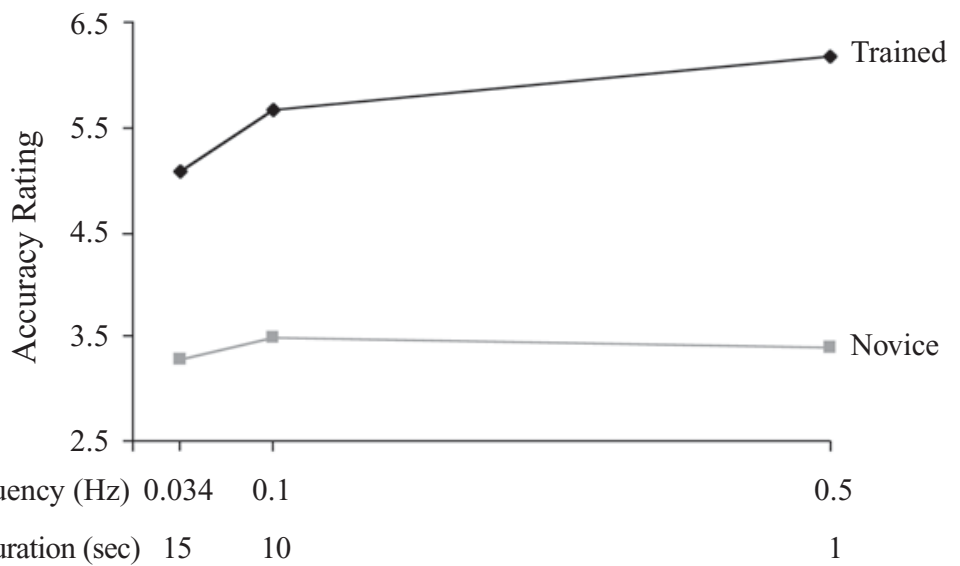

Alternation Duration (sec) $15 \quad 10$

Figure 6. Drawing accuracy as a function of gaze frequency (double labeled with light alternation duration) for novice and trained artists in Experiment 2. Higher ratings indicate more accurate drawings. 
artist's baseline accuracy. Experiment 4 was identical to Experiment 3, except that there were only two gaze frequency conditions: unregulated and $0.167 \mathrm{~Hz}$. The unregulated condition assessed the artist's baseline drawing accuracy. In the unregulated condition, the lights in both boxes remained on for the entire $10 \mathrm{~min}$, which allowed the artist to switch his or her gaze at will. The $0.167-\mathrm{Hz}$ frequency condition was used as the experimental condition because it was the highest gaze frequency in which both trained and novice artists could accurately follow the 3 -sec light alternations. Because the $0.167-\mathrm{Hz}$ frequency condition is lower than the gaze frequencies of most artists in Experiment 1, artists were predicted to draw less accurately in this condition than in the unregulated condition.

\section{Method}

\section{Participants}

Thirty-three nonart majors were recruited to participate as novice artists, and sixteen local artists were paid $\$ 10$ to participate as trained artists. Sixty nonart majors volunteered to participate as critics; 30 critics rated the accuracy of the novice artists' drawings, and 30 rated the accuracy of the trained artists' drawings. The critics had no formal training in the visual arts.

\section{Apparatus}

The two photographs of males used in Experiments 1, 2, and 3 and the apparatus used in Experiments 2 and 3 were used in Experiment 4.

\section{Procedure}

The artist's task. The artist's task was identical to that of Experiments 2 and 3, except that the gaze frequencies were $0.167 \mathrm{~Hz}$ and unregulated. The photograph $\times$ gaze frequency combination was counterbalanced, as was the order of presentation.

The critic's task. The critic's task was identical to that of Experiment 3 .

\section{Results}

To assess the effect of gaze frequency on drawing accuracy across artist training, accuracy ratings were subjected to a $2 \times 2$ mixed-model repeated measures ANOVA where artist was treated as a random effect (Cronbach's alpha; $\alpha=.93$ ). Because the trained and novice artists were rated separately, one cannot meaningfully compare the critics' mean rating of the trained artists with that of the novice artists. Therefore, the main effect of artist training was not tested. There was a significant effect of gaze frequency $[F(1,48)=9.71, p<.01]$, such that the drawings rendered in the $0.167-\mathrm{Hz}$ condition $(M=3.79, S D=1.11)$ were rated as less accurate than those rendered in which gaze frequency was not regulated $(M=4.27, S D=1.01)$. There was no significant interaction between artist training and gaze frequency $(F<1)$.

An analysis of the artists' gaze frequency in the unregulated condition indicates that trained artists switched their gaze more often $(M=0.32, S D=0.08)$ than novice artists $[M=0.24, S D=0.10 ; t(47)=2.92, p<.01]$. Importantly, both trained and novice artists' average unregulated gaze frequency was higher than $0.167 \mathrm{~Hz}$.

\section{Discussion}

The results of Experiment 4 confirm the hypothesis that the effect of gaze frequency demonstrated in Experiments 2 and 3 was the result of inhibiting artists' drawing accuracy. This result is consistent with the general finding of a positive relation between drawing accuracy and gaze frequency, because the $0.167-\mathrm{Hz}$ condition was lower than most artists' natural gaze frequency rates.

\section{GENERAL DISCUSSION}

The experiments in the present article assessed the relation between drawing accuracy and the rate at which artists glance between their drawing and the stimulus. Experiment 1 revealed a positive relation between drawing accuracy and gaze frequency. Experiments 2 and 3 demonstrated that gaze frequency influences drawing accuracy. Finally, Experiment 4 demonstrated that the effect of gaze frequency revealed in Experiments 2 and 3 was the result of inhibiting artists' drawing accuracy. In all four experiments, higher gaze frequencies were associated with more accurate drawings. Thus, the present experiments demonstrate that gaze frequency is an important contributor to drawing accuracy.

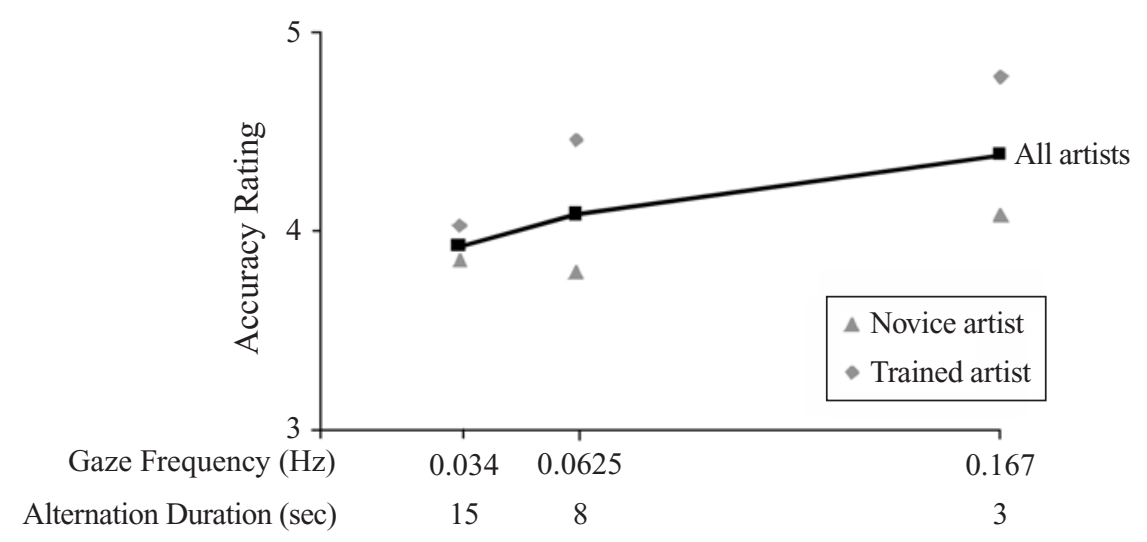

Figure 7. Drawing accuracy as a function of gaze frequency (double labeled with light alternation duration) in Experiment 3. Higher ratings indicate more accurate drawings. 
The results of the present experiments contradict the conclusions of Miall and Tchalenko (2001). Recall that Miall and Tchalenko proposed a negative relation between drawing accuracy and gaze frequency. Several factors may account this for discrepancy. First, Miall and Tchalenko did not test enough participants to assess a reliable relation. They compared one trained artist's eye movements with those of three novice artists, whereas the present experiment assessed over 130 artists in four experiments. Second, Miall and Tchalenko's participants had only $1 \mathrm{~min}$ to draw the portrait, whereas my participants had $10 \mathrm{~min}$ to draw the portrait. The 1-min time limit may have appeared short for the novice artists, and thus they may have hurried their drawings, accelerating all aspects of drawing, including their gaze frequency. In contrast, the trained artist had no time limit. One can only assume that Humphrey Ocean drew his portraits at a rate with which he was comfortable. In sum, although Miall and Tchalenko's intense case study of Ocean provides interesting insights into how he created portraits, their study cannot confidently make conclusions that address trends across artistic abilities.

Ballard et al.'s (1995) deictic system hypothesis predicts that high gaze frequencies lead to more accurate drawings. Recall that the deictic system hypothesis proposes that a system that is limited in WM capacity (e.g., humans) will compensate for this limitation by storing pointers to the location of perceptual information in the visual scene rather than storing the information directly. Such a system minimizes the use of WM by postponing the gathering of relevant perceptual information until it is required. Thus, a deictic system will break down if it is blocked from frequently gathering perceptual information.

When applied to the drawing process, a deictic system would frequently shift eye fixations from the to-be-drawn stimulus to the drawing. During each fixation, the system would gather only a small amount of perceptual information to be transferred to the drawing. Reducing the frequency of eye fixations would require the system to store more perceptual information in WM during each fixation. When this information exceeds the capacity of WM, the system would break down, and the accuracy of the drawings would be reduced. Indeed, Ballard and colleagues provide evidence for a performance decrease in a block-copying task when eye fixations are restricted and more than four blocks must be copied (Ballard, Hayhoe, Li, \& Whitehead, 1992).

Although the deictic system hypothesis predicts the positive relation between gaze frequency and drawing accuracy, it does not explain how drawing inaccuracies arise. If stimulus interpretation is a major influence on drawing accuracy, as the data suggest, gaze frequency likely modulates that influence. There are several possible mechanisms by which gaze frequency can modulate the influence of stimulus interpretation, none of which my data speak to directly. Indeed, it may be that the mechanisms by which gaze frequency influences the drawings of novice and trained artists are different. Nevertheless, in an effort to inspire future research, I present three of these mechanisms.
The first mechanism by which gaze frequency can modulate the influence of stimulus interpretation is through the use of ineffective mnemonic strategies. In accord with the deictic system hypothesis, gaze frequency may be negatively related to the amount of perceptual information one attempts to hold in WM. That is, artists with low gaze frequencies may attempt to hold more perceptual information in WM than artists with high gaze frequencies. Because WM's capacity is small, its limit is likely exceeded rather quickly. An artist with a low gaze frequency may therefore use a mnemonic strategy in an attempt to increase the amount of perceptual information he or she can store in WM. Ineffective mnemonic strategies will result in distorted memories. For example, one likely mnemonic strategy is to assimilate the to-be-drawn stimulus with prior knowledge of a prototype. ${ }^{6}$ Such a strategy will increase the effect of stimulus interpretation directly by merging the to-be-drawn stimulus with the prototype during encoding and/or indirectly through memory drift toward the prototype (e.g., Huttenlocher, Hedges, \& Duncan, 1991). In either case, the artist's knowledge of the prototype will unquestionably influence his or her rendering. In contrast, artists with high gaze frequencies likely store very little perceptual information on each fixation. Therefore, WM limits would not be exceeded, and mnemonic strategies would not be necessary.

A second mechanism by which gaze frequency can modulate the influence of stimulus interpretation is through the reduction of memory distortion. Werner and Diedrichsen (2002) provide evidence that spatial memory begins to distort within $50 \mathrm{msec}$ of the removal of the stimulus, and that this distortion increases as the time between the target and test stimulus increases. Furthermore, Huttenlocher et al. (1991) show that memory bias is a function of stimulus interpretation. In particular, the memory becomes biased toward the prototype of one's classification of the stimulus. Together, these findings suggest that the longer one holds spatial information in memory, the more that memory will become distorted toward the prototype. Artists with high gaze frequencies may take advantage of the graded effect of memory distortion by reducing the amount of time in which perceptual information must be stored. Thus, the faster the artist alternates his or her gaze between the stimulus and the drawing, the less time he or she will hold spatial information in memory, and therefore the less that spatial information will distort.

Although memory effects likely account for some of the variance associated with drawing accuracy, they probably do not account for all of it. There is abundant evidence that cognition influences perception even when the stimulus remains visible (e.g., Epstein, 1977; Rock, 1983, 1996). It is therefore likely that high gaze frequencies are evidence of a strategy to actively reduce the effect of stimulus interpretation at the time of encoding.

One strategy for reducing the effect of stimulus interpretation at the time of encoding is through the creation of a void viewing condition (Zeki, 1993, p. 229), in which only a very small portion of the stimulus is visible, thus 
minimizing context effects. For example, were one to look through a very small tube at a corner of a table, the gestalt "table" would have minimal influence on one's perception, whereas the retinal projection of the corner's angle would have maximal influence on one's perception. As a result, the effect of shape constancy would be reduced.

A third possible mechanism by which gaze frequency can modulate the influence of stimulus interpretation is through the creation of a virtual void viewing condition. Mack and Rock (1998) demonstrated that observers who focus their attention on part of a stimulus will not fully process stimuli that are away from their attentional focus. This finding is termed inattentional blindness because it describes a phenomenon whereby observers are "blind" to stimuli to which they do not devote attentional resources. Mack and Rock found that inattentional blindness is most influential when the stimulus is present for a fraction of a second. Longer presentation times result in more influence of the unattended stimuli. Thus, accomplished artists may actively inhibit context effects by creating a virtual void viewing condition through inattentional blindness. ${ }^{7}$ Artists with high gaze frequencies may successfully inhibit context effects, because short exposure times facilitate inattentional blindness. In contrast, artists with low gaze frequencies may not successfully inhibit context effects, because long exposure times inhibit inattentional blindness.

The present experiments beg the question, "Will forcing artists to increase their natural gaze frequency lead to more accurate drawings?" Although the data suggest that this may be the case, the present experiments cannot unequivocally answer this question. That is, Experiment 1 demonstrated that artists with high unregulated gaze frequencies drew more accurately than those with low unregulated gaze frequencies. Experiments 2, 3, and 4 demonstrated that gaze frequency influences drawing accuracy, but only for frequencies lower than the artists' unregulated gaze frequencies. To test the hypothesis that increasing an artist's natural gaze frequency will lead to more accurate drawings, one would have to develop a method to increase gaze frequencies to about $1 \mathrm{~Hz}$. One possibility is that over the course of training, artists learn (perhaps implicitly) to hasten their gaze frequencies. The present data provide some support for this hypothesis by revealing that trained artists' gaze frequencies are higher than those of novice artists. If one can train people to increase their gaze frequencies, research centering on this sort of training may be one method of successfully addressing this question.

\section{REFERENCES}

Ballard, D. H., Hayhoe, M. M., Li, F., \& Whitehead, S. D. (1992). Hand-eye coordination during sequential tasks. Philosophical Transactions of the Royal Society of London: Series B, 337, 338-339.

Ballard, D. H., Hayhoe, M. M., \& Pelz, J. B. (1995). Memory representations in natural tasks. Cognitive Neuroscience, 7, 66-80.

Blakemore, C. (1973). The baffled brain. In R. L. Gregory \& E. H. Gombrich (Eds.), Illusions in nature and art (pp. 9-48). New York: Scribner.

Blakemore, C., Carpenter, R. H. S., \& Georgeson, M. A. (1970).
Lateral inhibition between orientation detectors in the human visual system. Nature, 228, 37-39.

Cohen, D. J., \& BennetT, S. (1997). Why can't most people draw what they see? Journal of Experimental Psychology: Human Perception \& Performance, 23, 609-621.

Cohen, J., \& Cohen, P. (1983). Applied multiple regression/correlation analysis for the behavioral sciences. Hillsdale, NJ: Erlbaum.

DeReGowsKi, J. B. (1973). Illusion and culture. In R. L. Gregory \& E. H. Gombrich (Eds.), Illusions in nature and art (pp. 161-192). New York: Scribner.

DiAmOND, R., \& CAREY, S. (1986). Why faces are not special: An effect of expertise. Journal of Experimental Psychology: General, 115, 107-117.

EDWARDS, B. (1999). Drawing on the right side of the brain (2nd rev. ed.). New York: Penguin Putnam, Tarcher.

EPSTEIN, W. (1977). Stability and constancy in visual perception: Mechanisms and processes. New York: Wiley.

FreEMAN, N. H. (1980). Strategies of representation in young children: Analysis of spatial skills and drawing processes. London: Academic Press.

Freeman, N. H. (1987). Current developments in the development of representational picture-production. Archives de Psychologie, 55, 127-152.

GrEgORY, R. L. (1990). Eye and brain (4th ed.). Princeton, NJ: Princeton University Press.

HochberG, J. (1996). The perception of pictures and pictorial art. In M. P. Friedman \& E. C. Carterette (Eds.), Cognitive ecology (pp. 151204). San Diego: Academic Press.

Huttenlocher, J., Hedges, L. V., \& Duncan, S. (1991). Categories and particulars: Prototype effects in estimating spatial location. Psychological Review, 93, 352-376.

Kendall, R. (1993). Degas landscapes. New Haven, CT: Yale University Press.

Kozbelt, A. (2001). Artists as experts in visual cognition. Visual Cognition, 8, 705-723.

LEDER, H., \& BRUCE, V. (2000). When inverted faces are recognized: The role of configural information in face recognition. Quarterly Journal of Experimental Psychology, 53A, 513-536.

LEE, M. (1989). When is an object not an object? The effect of "meaning" upon the copying of line drawings. British Journal of Psychology, 80, 15-37.

MACK, A., \& Rock, I. (1998). Inattentional blindness. Cambridge, MA: MIT Press.

Miall, R. C., \& Tchalenko, J. (2001). A painter's eye movements: A study of eye-hand movement during portrait painting. Leonardo, 34, 35-40.

Murray, J. E., Yong, E., \& Rhodes, G. (2000). Revisiting the perception of upside-down faces. Psychological Science, 11, 492-496.

NaIRNe, J. S. (1996). Short-term/working memory. In E. L. Bjork \& R. A. Bjork (Eds.), Memory (pp. 101-128). San Diego: Academic Press.

Nunnaly, J. (1978). Psychometric theory. New York: McGraw-Hill. ReITH, E. (1988). The development of use of contour lines in children's drawings of figurative and nonfigurative three-dimensional models. Archives de Psychologie, 56, 83-103.

Rock, I. (1983). The logic of perception. Cambridge, MA: MIT Press.

Rock, I. (1996). Indirect perception. Cambridge, MA: MIT Press.

Rosenberg, J. D. (1963). The genius of John Ruskin. New York: Braziller.

SzE, M. (1956). The way of painting. New York: Vintage.

TANAKa, J. W., \& SengCo, J. A. (1997). Features and their configuration in face recognition. Memory \& Cognition, 25, 583-592.

THouless, R. H. (1932). Individual differences in phenomenal regression. British Journal of Psychology, 22, 216-241.

Van Sommers, P. (1984). Drawing and cognition: Descriptive and experimental studies of graphic production processes. Cambridge: Cambridge University Press.

WERNER, S., \& DiEDRICHSEN, J. (2002). The time course of spatial memory distortions. Memory \& Cognition, 30, 718-730.

Willats, J. (1997). Art and representation. Princeton, NJ: Princeton University Press.

ZEKI, S. (1993). A vision of the brain. Oxford: Blackwell. 


\section{NOTES}

1. D. J. Cohen and Bennett (1997) make a distinction between two types of misperceptions: illusions and delusions. Illusions cannot be overcome through careful observation alone, and therefore everyone is vulnerable to them. Because illusions are relatively rare, they likely are not the cause of the majority of drawing errors. Delusions, on the other hand, are false beliefs that are held despite invalidating evidence, and which, therefore, can be overcome with careful observation. Delusions arise from stimulus interpretation. For example, when drawing a face, one may draw an ellipse for the head because he or she "knows" that heads are elliptical. Unlike illusions, one would not want to claim that the artist cannot tell the difference between an ellipse and a face. The errors present in Lee's (1989) experiment were likely the result of delusions.

2. If stimulus interpretation is the major source of drawing errors, and it had an equal impact on the critique and rendering processes, artists would be unable to recognize that their drawings are inaccurate. Experiment 4 of D. J. Cohen and Bennett (1997) demonstrated that artists recognized that their drawings were not accurate, yet they were unable to correct the errors. This suggests that stimulus interpretation has a greater impact on the rendering process than on the critique process.

3. Two of the variables, critics' rating and photo/drawing ratio, significantly deviated from normality (Kolmogorov-Smirnov test; $p<.05$ ). To assess whether distributional skew affected the results, I transformed these variables into normality and reran all tests. The pattern of results was identical.

4. Because photo/drawing ratio had no effect on drawing accuracy in Experiment 1, I decided to make the switch rate constant. Although it is conceivable that a different ratio may influence drawing accuracy, it is unlikely that it would influence the direction of the effect. That is, artists may draw a bit more or less accurately with a different photo/drawing ratio, but it is highly unlikely that gaze frequency will become irrelevant or that the effect would be reversed.

5 . The critics' ratings of the novice artists were positively skewed (Kolmogorov-Smirnov, $p<.05$ ). To assess whether the lack of normality accounted for the results of the novice artists, I normalized their data and reran the simple effects on their data. The pattern of results is identical to that of the raw data.

6. I do not provide an exact description of the prototype, because artists may assimilate the to-be-drawn stimulus with one of many possible prototypes (e.g., a prototypical semantic representation of the to-bedrawn stimulus, prototypical graphical tokens that represent features of the to-be-drawn stimulus, a prototypical drawing of the to-be-drawn stimulus). For example, novice artists may assimilate the to-be-drawn stimulus with prototypical graphical tokens that represent features of that stimulus. In contrast, trained artists may assimilate the to-be-drawn stimulus with a prototypical drawing of that stimulus. In both cases, as gaze frequencies decrease, the influence of the prototype will increase (perhaps during encoding and/or through memory drift toward the prototype). Nevertheless, the renderings created by the trained and novice artists would appear quite different because of the different prototypes employed.

7. Although the deictic system provides a pointer to the visual information, the artist still must view and render the stimulus. The virtual void viewing ability will help the artist view the visual information (to which the deictic system points) while simultaneously inhibiting interpretation.

(Manuscript received March 8, 2004; revision accepted for publication November 22, 2004.) 